Leprosy is so far-reaching in its social results, for the individual affected is cut off to such an extent from his fellow beings as to render his earning a livelihood very precarious, that whether we look at the matter from the standpoint of humanity or from that of sound policy, it is seen to be the duty of every Government to prevent its further spread.

It does not seem to have been sufficiently understood that all measures of preventive medicine which are based on sound scientific principles are excellent business propositions. I believe that the money invested in the study of Malta fever, now that the cause has been found, is saving the Government yearly a sum which would be sufficient to pay a dividend of 100 per cent. or more.

Such examples could easily be multiplied; one which lately came under my notice in Russia deserves mention here. Relapsing fever in Moscow alone attacked about 1,500 to 3,000 individuals each winter. Each patient remained in hospital from three to five weeks. Since the invention of salvarsan, which is a wonderful specific for similar diseases of the blood, the stay has been shortened to five days or a week. The resulting saving is about 30,000 to 50,000 roubles $(£ 3,000$ to $£ 5,000)$ a year!

The sanitation of Khartoum, which owes so much to the work of Dr. Andrew Balfour, lately Director of the Wellcome Laboratories at the Gordon College, has also made itself pay over and over again in hard cash by the resulting influx of visitors and saving in hospital expenses for inliabitants and officials.

How are these comforting examples applicable to the problem of leprosy in the British Empire? By a system. atic and persevering attempt to get rid of the disease by instituting settlements for the voluntary segregation of the worst cases, where the numbers are too great to be dealt with at one time, as is the case in India; and by a system of universal segregation in properly conducted asylums in those colonies and dependencies where relatively small numbers only are to be accounted for. Unfortunately, in some countries the principle "out of sight out of mind" is still applied to the leper asylums, and money spent on this principle is almost wasted. It should, however, be clearly understood that every system of segregation needs the cooperation of the lepers themselves, and this can only be obtained by paying the asylum staffs proportionately and adequately, and securing proper medical attendance.

The experience of other countries shows us that such a course would lead to the gradual extinction of leprosy, a disease which has now been known for so long a time that its survival should no longer be tolerated.

\section{REMARKS ON ACUTE PANCREATITIS.} WITH NOTES OF SEIEN CASES.

A. J. BLAXLAND, M.S., F.R.C.S.,
ASSISTANT SURGEON, NORFOLK AND NORWICH HOSPITAL; AND

G. P. C. CLARIDGE, M.B., B.S., HONORARY PATHOLOGIST AND BACTERIOLOGIST, NORFOLK AND NORWICH HOSPITAL.

JUDGING from the literature on acute pancreatitis, one would imagine this condition, although very serious, to be one in which, with surgical intervention, a cure may be expected in about 40 per cent. of the cases.

We have recently had under observation 6 cases of this condition, all of which terminated fatally; 4 of these were treated by operation and 2 died undiagnosed. Although we are aware that the number of cases is small, our impression, gained from a study of them, is that the condition is more serious than one would be led to suppose by the cases already published. We may have been unfortunate in having to deal with a specially virulent set of cases, but we cannot help thinking that, as a rule, unsuccessful results are not published, and that this mitigates against a proper idea of the great gravity of the condition.

CaSE I.

A male, aged 40, was admitted to the Norfolk and Norwich Hospital on December 22nd, 1909, with the history that the illuess started suddenly on December 20th with acute pain in the abdomen and vomiting. The pain continued severe and the vomiting became profuse. There was a history cf previous attacks of biliary colic.

\section{Operation.}

On admission the abdomen was tender and rigid. No distension; no dullness on percussion. General condition ver bad, skin cold and clammy. Operation two hours after admission-supra-umbilical laparotomy. Pancreas found hard, en larged, sloughing in places, and beads of pus. Tubes inserted after incising the gland above the stomach and below. A few glistening white patches of fat necrosis seen in transverse mesocolon; none in omentum. The patient died twelve hours after operation.

\section{Necropsy.}

Closed wound above umbilicus, middle line, $5 \mathrm{in}$. in length, and an open wound $1 \mathrm{in}$. in length below the umbilicus. General peritonitis, lymph being found on the peritoneum Fat necrosis in the transverse mesocolon. Three gall stones in the common bile duct, the gall bladder being full of stones. The tail of the pancreas quite necrotic, the head and body being of a dark red colour, swollen and hard.

Microscopical Examination.-The architecture of the pancreatic lobules is well marked. In some the cells are loose, breaking away from each other and the basement membrane, others are completely necrotic; in others abscess formation is taking place in the middle of a lobule already necrotic; others again have become abscess cavities. Extensive haemorrhage has taken place, mostly intralobular. The ducts in most cases show little change, but those in the centre of the larger necrotic areas are necrotic as well; in these the cell outlines have quite disappeared. Areas of fat necrosis are abundant. The islands of Langerhans are not to be distinguished in the necrotic areas.

CASE II.

A male, aged 40 , was admitted to the hospital at 8.45 p.m. on August 27th, 1910. The illness began on the night of August 24th with sudden pain on the right side of the abdcmen and vomiting; the latter became constant and profuse. On admission the patient was in a very collapsed condition; abdomen rigid and distended, dullness over pubes extending into right loin.

Operation.

At 10 p.m. on August 27th anaesthesia was induced with a mixture of ether and chloroform. The stomach was washed out preliminary to the operation, and a quantity of filthy brown fuid evacuated. Median subumbilical laparotomy performed reddish dirty fluid found in the pelvis; small intestine very dis tended and dark red; circular, glistening white areas of fat necrosis seen in mesentery, but none in the patient's condition was so bad that no attempt could be made tc explore the pancreas, and he died from failure of respiration while the wound was being closed.

\section{Necropsy.}

A little reddish dirty fluid was found in the abdomen and fat necrosis in the mesentery. The pancreas was found firm and swollen, and appeared to be full of haemorrhagic effusion. The liver was somewhat enlarged and the gall bladder was distended, several stones being present, but the ducts were all patent. Nothing else abnormal was found, except old pleural adhesions on the right side.

Microscopical Examination.-The lobules in places appear normal, but most of them are in a condition of early necrosis, the cells being dislocated from their normal positions, and some parts have become quite necrotic. There is some inflammatory reaction, with haemorrhage around the pancreas. There are also areas of fat necrosis within the substance of the gland. The islands of Langerhans have suffered less than the rest of the organ.

CASE III.

A female, aged 51, was admitted to the hospital on November 19th, 1910. The illness began twenty-four hours before admis sion with sudden violent pain in the abdomen. History of "indigestion" for over a year, with two attacks of haematemesis. On admission the patient was in great pain, vomiting frequently. Abdomen rigid and tender, especially in the upper part; slight dullness above pubes. Very collapsed; no pulse to be felt at the wrist. Dr. Long and Dr. Cleveland saw the patient with one of us, and although a definite diagnosis was not made, it was obvious that an operation held out the best chance of life. At their suggestion the patient's limbs were firmly bandaged and two pints of saline and some pituitary extract were administered prior to the operation.

\section{Operation.}

Suprapubic laparotomy was performed. Dirty blood-stained fluid was found in the pelvis, and the omentum showed obvious patches of fat necrosis. The abdomen was washed out with saline, an incision made in the left loin, and a tube passed through to the tail of the pancreas, which was felt to be hard and enlarged.

November 20th. Patient comfortable; no pain; pulse, however, not perceptible. A quantity of dirty fluid draining through the tube.

November 21st. Pulse perceptible.

November 22nd. Patient better, pulse stronger, respirations , temperature subnormal.

The improvement did not continue after the $23 \mathrm{rd}$, and the patient died on the 28 th. 
Necropsy.

I fat necrosis well marked in omentum, mesentery, and the fat under the parietal peritoneum; two gall stones in the gall bladder, the duct not obstructed; the pancreas greatly swollen, and appeared haemorrhagic; stomach dilated, a small erosion near the ampulla of Vater.

Microscopical Examination.-The gland lobules show the changes of early necrosis, the ducts and islands of Langerhans take part in the same change. Areas of fat necrosis are abundant.

CASE Iv.

A male, aged 34, was seized with sudden pain in the abdomen and sickness on September 9th, 1911. He was seen by Dr. Aldred of Wroxham, who sent him to the Norfolk and Norwich Hospital on the 11th. On admission his abdomen was unif rrmly distended, and there was resistance in the left loin. There was no collapse.

Operation.

An enema was given with slight result, but as the pulse-rate was increasing operation was decided upon. Thirty-six hours after the onset of the attack laparotomy was performed through the left rectus, above the umbilicus and extending 1 in. below The peritoneal cavity was found to contain dirty-red fluid, and there were obvious patches of fat necrosis scattered all over the cmentum. An opening was made through the great omentum just below the stomach with blunt forceps. The pancreas, which was much swollen and very tense, was incised with forceps and a drainage tube and gauze wick passed down. The gall bladder was distended but no stones could be felt.

After-History.

The day after the operation the patient was better, slightly jaundiced. The wound was discharging profusely. The improvement was maintained until September 17th (sixth day after operation), when the pulse began to get quicker and there was increased resistance in the left loin. An incision was made under eucaine in the left loin and a little dirty fluid evacuated and a drainage tube inserted.

On the 20th the laparotomy wound was gaping owing to digestion from the discharge, and silk sutures were inserted

The patient gradually got weaker and died from exhaustion on September 27 th-that is, sixteen davs after operation.

$$
\text { Necropsy. }
$$

Well-nourished man with a large gaping wound in his abdomen. A little clear fluid found behind the omentum. All the abdominal fat thickly studded with fat necrosis. Above the transverse colon a track leads down to the pancreas. At the bottom of this track is a mass of necrotic tissue and pus. The head of the pancreas appeared to be normal, but the body and tail were absolutely necrotic, the whole organ floating free. The gall bladder contained no stones, but was firmly adherent to the small intestine. There was no stone found in any of the ducts.

Microscopical section of the head of the pancreas showed that it was in a condition of early necrosis with a very little haemorrhage into the substance of the gland. The islands of Langerhans were normal. Large areas of fat necrosis were present. The tail of the organ was found on section to be absolutely necrotic.

\section{CASE V.}

A woman, aged 48 , said to have suffered from ulcerated stomach three and a half years before, and since then to have enjoyed good health, complained of abdominal pain on the evening of September 26th, 1911. The next morning she felt better, and did not consider it necessary to see a doctor. The husband left home at 7.30 a.m. for work, and, returning at 6.30 p.m., he found her dead, lying face downwards on the floor. A chair that he had placed beside the bed was knocked over, and there was dried vomit found on and by the bed.

\section{Necropsy.}

On opening the body fat necrosis was found to be present in the subperitoneal fat as well as in the omentum. The pancreas was found surrounded by effused blood and was hard and swollen; on section it was seen to be full of effused blood. The gall bladder contained four gall stones.

Microscopical section showed that the organ was completely necrotic, although the architecture of the gland could still be made out. There was a large amount of interstitial haemormade out. There was a large amount of interstitial haemor-
rhage and many areas of fat necrosis. There were no inflammatory cells present.

\section{Case vi.}

A man, aged 54, who had never had any serious illness, but had suffered from occasional vague abdominal pains for ten or twelve years, consulted Dr. Starling on December 26th, 1910, who treated him for indigestion. He improved steadily up to January 14th, 1911, when he had an attack of pain in the abdomen, which returned early in the morning of January 15th. When seen at 8.30 a.m. he was obviously very ill, face ashy colour, nose cyanosed, pulse 90 and weak, anxious drawn expression, he had vomited once. He rapidly got worse and died at 7.45 p.m. that day.

$$
\text { Necropsy. }
$$

A little dirty fluid was found in the peritoneal cavity. There was some haemorrhagic effusion around the pancreas which was swollen and firm. On section it appeared to be full of effused blood. One large gall stone was found in the gall bladder. No fat necrosis was seen.

Microscopical section of the pancreas showed that there was a good deal of fibrous tissue laid down between the lobules of the gland. The parenchyma of the gland was completely necrotic, a cell here and there only showing a nucleus, and this in a state of degeneration. There was diffuse general haemorrhage into the gland and many areas of fat necrosis, with only slight inflammatory reaction.

\section{Pathology.}

The intimate anatomical association of the common bile duct and the pancreatic duct has been considered the important factor in the causation of this condition, the actual cause being the invasion of the pancreatic duct by bile or duodenal contents. This theory is based on the frequent presence of gall stones in these cases, the passage of a gall stone either diverting the bile into the pancreatic duct or so dilating the orifice as to allow duodenal contents to enter the now incompetent valves (of the ampulla of Vater). ${ }^{2}$ Our cases strengthen this view, as in five out of six gall stones were present, and in the other there was evidence of old inflammatory trouble, possibly due to the former presence of gall stones.

Our opinion is that this condition is caused by the entrance of bile or duodenal contents into the pancreatic duct, which there activates the pancreatic ferment and causes a digestion of the gland, a circumstance which may be followed by the invasion of micro-organisms. In some cases this digestion apparently takes place without any invasion by micro-organisms. This digestion in the gland affects the blood vessels, leading to haemorrhage in and around the gland. This process then spreads beyond the confines of the gland, leading to the phenomenon of fat necrosis in other parts of the abdomen. It appears that fat necrosis is not seen in cases in which pancreatic juice alone has found its way into the peritoneal cavity, but that when this is mixed with bile the phenomenon occurs. The above theory of pancreatitis seems to us to be borne out by the pathological findings in these cases, in which all stages of the condition were met with, from the very earliest, in which the cells still stain fairly well but are seen to be separated from each other and from the basement membrane, to the most advanced, in which all indication of the structure of the gland has disappeared and only a few nuclear fragments are seen. These condi. tions, we maintain, are due to the invasion of the gland by bile or bile-containing fluid by way of the ducts. In some of these cases, too, we see inflammatory reaction and abscess formation in addition to the above features, and in these cases we suggest the gland has been secondarily infected by some micro-organism. If this infection does not take place, no inflammatory reaction is seen in the gland at all, and the case is not one of pancreatitis but pancreatic necrosis. The very acute symptoms observed in these cases is due, we suggest, to the absorption of toxic products of the digestion going on in the gland.

\section{Diagnosis.}

All are agreed that the diagnosis of this condition before laparotomy is extremely difficult.

As regards history, though five of the patients had gall stones, only one gave a history of biliary colic or jaundice (Case I). Three cases (III, v, and vi) had had bad "indigestion" for over a year with or without haenatemesis, which made one suspect perforated gastric or duodenal ulcer. In all the cases the condition started suddenly with pain and vomiting, the latter being frequent and copious, as is usually noted.

In all the abdomen was rigid and tender; in one case (No. III) the tenderness was most noticeable in the upper. part of the abdomen, and in one case (Iv) in the left loin. In Cases II and III there was obvious dullness on percussion above the pubes.

An extreme degree of collapse was a marked feature in four of the cases. In Case III the patient was pulseless at the wrist within twenty-four hours of the onset, and remained so for twenty-four hours after the operation, when she began to improve. Cases v and vi ended fatally within twenty-four hours of the onset of acute symptoms.

In our opinion the extraordinary amount of collapse is the only symptom which would suggest the diagnosis; but in some cases, probably of a less acute nature, assistance may be obtained by finding a localized resistance in the upper part of the abdomen. 
TreatMent.

In acute abdominal conditions with sudden onset it will generally be admitted that, whether a definite diagnosis has been made or not, surgical intervention holds out the best chance of recovery. In this condition death is almost certain to ensue unless the process of necrosis is arrested by incising the gland and free drainage; this should be performed as soon after the onset as possible; delay is fatal. That operation may be successful is proved by the published cases.

After performing a laparotomy a definite step in the diagnosis is made if dirty red-brown fluid is found in the pelvis, which was a marked feature in Cases II, III, and IV. If there is fat necrosis in the omentum the diagnosis is fairly obvious; but fat necrosis, although present in all the cases operated on, occurred in the omentum in only two of them (Cases III and IV); in Cases I and II it was present, at the time of the operation, only in the mesentery of the small intestine and in the transverse mesocolon. Finally, the pancreas will be found to be greatly enlarged, and the question arises whether one should be content with simple drainage of the pancreatic region, or whether cholecystostomy should be performed. One should be guided, we consider, chiefly by the general condition of the patient. As a rule, any prolongation of the operation should be avoided.

As a preliminary to the operation, we think it advisable to wash out the stomach, as in other conditions in which frequent and profuse vomiting is a feature. Measures to combat the marked collapse should also be taken prior to the operation, ihe most reliable, after warmth, being saline infusion and the exhibition of pituitary extract.

- Since writing the above we have had the opportunity of secing another case of this disease:

CASE VII.

A male, aged 47, who for several years had suffered with occasional attacks of pain in the epigastrium which had no relation to food, but had never been jaundiced, at 3 a.m. on May 6th, 1912, was suddenly seized with severe pain in the epigastrium, and vomited once. He was admitted to the thorich Hospital this his bowels were open. He was then collapsed and cranosed, with rapid, feeble pulse. There was no movement of the upper abdomen on respiration, slight tenderness on pressing over the epigastrium, abdominal muscles rigid, and marked dullness in both flanks. The liver dullness was not obliterated.

\section{Operation.}

He was given 3 pints of saline and 1 c.cm. of pituitary extract by infusion during the operation. Mr. E. W. Everett performed suprapubic laparotomy and found blood-stained fluid in considerable quantity in the peritoneal cavity; he inserted a tube into the pelvis and then opened the abdomen above the umbilicus. On lifting up the omentum, fat necrosis was seen and the gall bladder was found to be full of stones. The latter was opened and drained, and the supraumbilical wound was closed. The patient was returned to bed in the Fowler position closed. The patient was returned to bed in the Fowler position He died at 10 p.m.

Necropsy.

A well-nourished, muscular man. General peritonitis, with dirty blood-stained fluid in the peritoneal cavity. Fat necrosis in the great omentum, appendices epiploicae, mesentery, and on the under surface of the diaphrasm-most marked in the region of the spleen. The pancreas : head and first part of the body dark red in colour and of the consistency of soft indiarubber: the rest of the body and the tail appeared normal. The bile ducts were patent and apparently normal. The pancreatic duct was apparently normal, and was not bilestained.

Microscopical sections show that the head of the pancreas is completely necrotic, no sign of the gland substance remaining; this part of the gland is extensively infiltrated with blood. The tail of the organ is seen to be in the first stage of necrosis, but no haemorrhage has taken place here. Between these two points all stages of necrosis are found. Throughout the organ there are areas of fat necrosis, but nowhere is there seen any evidence of inflammatory reaction.

In conclusion, we wish to record our thanks to Dr. H. J. Starling and Mr. E. W. Everett for their kind permission to use the notes of the cases under their care.

REFERENCES.

${ }^{1}$ Moynihan, Keen's Surgery, vol. iii. ${ }^{2}$ Opie, Disease of the Pancreas.

THE Middlesex Hospital and the New Hospital for Women each receives a bequest of $£ 2,000$ under the will of the late Mr. John Anderson, of Porchester Terrace, W.

\section{THE DIAGNOSIS AND TREATMENT OF INTESTINAL OBSTRUCTION.*}

BY EDWARD DEANESLY, F.R.C.S., HONORARY SCRGEON, WOLVERHAMPTON GENERAL HOSPITAL.

Excessive Mortality.

IT is a reproach to our profession that many hundrods of men, women, and children die every year from intestinal obstruction due to mechanical causes. It is no exaggeration to say that in every case life could be saved or prolonged by surgical operations performed at an early stage of the illness. The mortality of cases of acute obstruction of the bowels submitted to operation is still appalling, probably as much as 75 per cent., and this is entirely due to the large number of cases in which surgical aid is sought or accepted as a last resonrce, although it is obvious that mechanical obstruction can only be removed by mechanical means. This deplorable and unnecessary waste of life is mainly due to the continuance of the old tradition which assigned all internal diseases to the province of the physician, or' as the Germans put it, of "inner Medicine."

An Essextially Surgical Condition.

No modern physician would, of course, deny that the treatment of mechanical obstruction of the bowels is wholly and solely a surgeon's job. $\mathrm{H}_{3}$ is justified, of course, in pointing out that it is sometimes a matter of great difficulty to decide whether a given patient has or has not obstruction of the bowels, and whether, the fact of obstruction being admitted, it may not be due to some cause capable of spontaneous disappearance. Influenced, however, by such doubts, the tendency of the physician is to wait and see. No doubt most problems are eventually solved by this method, but when the solution is so often made at the expense of the patient's life, it would seem a speedier and safer way to open the belly and look.

I do not suggest that medical practitioners are wholly to blame for the late stage at which the majority of cases of intestinal obstruction reach the hands of the surgeon. The ancient habits and beliefs of medicine continue to be cherished by the public long after they have been discarded by the profession, and many patients still abstain from seeking medical advice until they have treated them. selves on orthodox lines by purgatives, enemata, and poultices without relief. Too often, nevèrtheless, when the practitioner is called in attempts are made to continue this futile treatment instead of making a definite diagnosis, or advising an exploration.

Confusion between Constipation and Obstruction.

The reluctance both of the practitioner to advise and of the patient to accept an immediate or early operation is due in no small measure to an babitual confusion between the ideas conveyed by the terms "constipation" and "obstruction." The term "obstruction" is generally, and should be always, used to mean actual mechanical obstruction to the lumen of the bowel sufficient to hinder or actually arrest the onward flow of the intestinal contents at a particular point. Obstruction so defined, if it exists, is obviously a surgical condition, curable only by surgical means, and surgically urgent in proportion to the acuteness and degree of the obstruction.

The term "constipation," on the other hand, means nothing more than some greater or less delay in the periodical evacuation of the bowels. It is quite common to meet with persons who habitually go several days without an evacuation, and not very uncommon to sce a patient who has had no evacuation for as much as a week or more without showing any signs of acute illness or even discomfort, and without obstruction of the bowel at any point. It is true that this delayed progress of the intestinal contents, or intestinal stasis, is often associated with certain anatomical changes in the large intestine, and that it has been attributed by Sir Arbuthnot Lane and others to certain sharp bends or kinks caused by the dropping of certain parts of the colon, so that it might be described as a form of organic intestinal obstruction. Whether we accept Sir Arbuthnot Lane's theory or not-

* A paper read before the Staffordshire Branch of the British Medical Association. 